\title{
Optimal Education Plan of Employees Using Maintenance Model
}

\author{
Shigeshi Yamashita \\ Department of Engineering, Tottori University, \\ Minami 4-101, Koyama, Tottori-Shi, Tottori, 680-8552, Japan. \\ E-mail: shigeshi_yamashita@mhi.co.jp \\ Kodo Ito \\ Department of Engineering, Tottori University, \\ Minami 4-101, Koyama, Tottori-Shi, Tottori, 680-8552, Japan. \\ Corresponding author: itokodo@ tottori-u.ac.jp \\ Sho Kawakami \\ Department of Engineering, Tottori University, \\ Minami 4-101, Koyama, Tottori-Shi, Tottori, 680-8552, Japan. \\ E-mail: B17T4038H@edu.tottori-u.ac.jp \\ Truong Dinh Anh Khoa \\ Department of Engineering, Tottori University, \\ Minami 4-101, Koyama, Tottori-Shi, Tottori, 680-8552, Japan. \\ E-mail: B17T4061Y@edu.tottori-u.ac.jp
}

(Received on February 10, 2021; Accepted on May 30, 2021)

\begin{abstract}
The employee education is indispensable for companies to improve productive efficiency and product quality. In general, the employee education is divided into two types, i.e., On-job trainings and Off-job ones, and Off-job trainings are divided into two types, i.e., compulsory educations and non-compulsory ones. Compulsory educations such as safety program and compliance education, are necessary to maintain daily production without any accidents. Although all employees of a department and a division gather in a classroom annually in conventional compulsory educations, daily e-learning education complements and strengthens conventional compulsory ones today because employees forget what they learn by annual learning. In past studies, the logit model is used for modeling the influence from education receipt to its memory condition and quantitative relationship between the effect of traffic safety education and the accident-related humanerrors was clarified. The effectiveness of the safety driving educational program was indicated by Structural Equation Modelling method. In this paper, an annual compulsory which is complemented and strengthened by e-learning, is discussed. The expected cost rate of education is expressed using imperfect maintenance models and optimal policies which minimizes it is considered. Although we use the exponential function which denotes the occurrence probability of accidents which are caused by forgetting lessons, the actual occurrence probability which is approximated from the actual data would be a complicated one. For solving such complex optimization problems, metaheuristic methods can be applied.
\end{abstract}

Keywords- Vocation education training, E-learning, Education plan, Imperfect maintenance, Optimal policy.

\section{Introduction}

In the international competition, companies have to improve their performance, and the employee education is one of effective methods for the purpose because it has a positive impact on the performance of individuals and teams (see Aguinis and Kraiger, 2009). There exists a positive relation between the education investment and the organizational performance (see Aragon and Valle, 2013). 
The employee education has three methods, i.e. on-the-job training, off-the-job one, and on-the-job and off-the-job one (see Maršíková and Šlaichová, 2015). In case of on-the-job trainings, elder employees coach younger ones and employee education are provided through jobs. In case of offthe-job trainings, employees are gathered at the training center and are lectured. On-the-job and off-the-job training are different from the other two methods and an example of that is e-learning.

The employee education is divided into two categories, i.e., the initial vocational education training (IVET) and the continuous vocation educational training (CVET) (see Cedefop, 2008, 2011). IVET is the education which is undertaken at joining the company and at starting new specialty jobs after joining. CVET is the education which is undertaken for updating knowledge and skills of employees. Most representative manufacturing companies in Japan such as Mitsubishi Heavy Industries, Ltd. and Toshiba have provided a lot of off-the-job IVETs for mechanical, electronic, software, and project engineers who are athirst for the innovative knowledge and skills (see Harashima et al., 2006; Wani, 2007). Safety and compliance trainings are undergone in companies as off-the-job CVETs because they are necessary to avoid fatal accidents during production and legal troubles during business.

To prevent a fatal accident, the safety pyramid shows that dozens of minor injuries and hundreds of safety incidents with limited or no damage have to be measured (see Gnoni and Saleh, 2017; Heinrich, 1931; Manuele, 2011; Masimore, 2007). Minor injuries and safety incidents with limited or no damage are called near misses and such accident case studies are lectured to employees as off-the-job CVET (see JISHA, 2006). Such lecture has to be performed periodically to prevent forgetting and have to be performed for all employees concerning production, i.e., managers, engineers, fabrication and assembling workers of the production section. Because new fatal accidents happen and hundreds of near misses are reported annually, the contents of the lecture are renewed annually.

Although the Kirkpatrick four-level approach is utilized widely as the training qualitative evaluation model among practitioners, companies do not evaluate quantitative (economic) effects of training because the investment of employee education is relatively low compared to other investments in companies and the quantitative evaluation of training is practically difficult (see Arago'n-Sa'nchez et al., 2003; Kirkpatrick, 1996; Smidt et al., 2009). Although the investment of employee education is relatively low in company, it may become a heavy burden in some section of a company. Because production work processes cannot be automatized and lots of fabrication and assembling workers are necessary in case of aerospace industries, the off-the-job CVET cost for them causes financial loss for production sections of aerospace companies.

Today, on-the-job and off-the-job training such as e-learning is used for declining education cost because developing cost of e-learning program is much cheaper than off-the-job training in production sections. In case of Mitsubishi Heavy Industries, Ltd., employees solve 5 questions on their PC every day, their scores are registered automatically, and evaluation results of all employees are reported weekly, monthly, and annually to their boss (see Wani, 2007). Because these questions are selected randomly from the 6500 questions database and employees repeatedly solve 6500 questions in a certain period, such e-learning is a smart method to refresh a large amount of significant knowledge periodically.

Although forgetting certain accident case study causes accidents which relates to the case study, forgetting the case study does not always cause any accidents immediately because an accident 
occurs when different types of events occur at the same time and this is well known as the Swiss cheese model (see Reason, 2000). There exist stochastic relationships between forgetting accident case studies and real accidents and it is acceptable that occurrence probabilities of accidents increase when the amount of forgotten accident case studies grows. Both physical human phenomena such as fatigue and recovery and psychological phenomenon such as learning and forget are represented by the exponential distribution (see Elshamy, 2002; Jaber et al., 2013).

The logit model is used for modeling the influence from education receipt to its memory condition (see Armstrong and Sloan, 1989) and Shi et al. (2010) clarified the quantitative relationship between the effect of traffic safety education and the accident-related human-errors by applying the logit model and analyzing data 384 commercial vehicle drivers in China. Because safety education methods affect the reduction of traffic accidents was reported, effective methods are needed for the forceful safety education (see Hickman and Hanowski, 2011; McGehee et al., 2007). Wang et al. (2018) revealed that three behavior-based safety education methods, including monthly one-on-one face-to-face meetings with video education, could reduce traffic accidents significantly by referring the negative binomial model and resolving data of 34 commercial vehicle drivers in China. Because identifying important human errors and violations is important for developing efficient trainings and education programs of safety driving, Driver Behavior Questionnaire (DBQ) was developed in Britain (see Reason et al., 1990). Topolšek et al. (2019) indicated that the effectiveness of a Slovenian safety driving education program by using Structural Equation Modelling (SEM) of 183 Slovenian drivers DBQ data. Although the effectiveness of safety educations was clarified by statistical analysis of data which were actually obtained in these studies, mathematical models which determine the education interval is necessary for designing new education plans. In this paper, the employee safety education which contains periodical off-the-job CVET and daily elearning is considered and optimal education plans using maintenance models which minimize economical investment are discussed.

\section{Model 1}

Although accidents can be avoided just after receiving off-the-job CVET, their occurrences increase with time and safety knowledge might decrease gradually by them. Such situations are very similar to the increment of system failures in reliability models. So that, using the imperfect preventive maintenance (PM) model (Nakagawa, 2005), the increment of accidents after off-thejob CVET can be modelled.

We make the following assumptions for Model 1, which is the fundamental model with both effects of off-the-job CVET and daily e-learning:

(i) A safety training as the off-the-job CVET is held periodically for engineers in production section. To prevent to forget lessons which are learned at the training, the exercise with volume $T(0<T<\infty)$ of e-learning is practiced every day, and when the total volume is $N T(N=1,2 \ldots)$, the training ends. Because volume $T$ is proportional to time of training, we use time $T$ in place of volume $T$.

(ii) Some accidents related with case studies by forgetting lessons occur during the training. When the exercise volume $T$ is the function of time, its occurrence rate is given by $h(t)$ at time $t$, which remains undisturbed by any accidents and increases strictly with time $t$.

(iii) The rate of forgetting lessons can be slowed down with the number of trainings, which is proportional with time $t$. By every exercise, the training time reduces to at $(0<a \leq 1)$ when it was $t$ without exercise, i.e., its time becomes $t(1-a)$ units of time smaller at 
every exercise.

(iv) Cost of each accident fee is $c_{1}$, cost of making a whole exercise is $c_{2}$, and cost of the safety training is $c_{3}\left(c_{3}>c_{2}\right)$.

The expected cost rate $C_{1}(N, T ; a)$ is, from (see Nakagawa, p178, 2005),

$$
\begin{aligned}
C_{1}(N, T ; a) & =\frac{1}{N T}\left[c_{1} \sum_{j=0}^{N-1} \int_{A_{j} T}^{\left(1+A_{j}\right) T} h(t) d t+(N-1) c_{2}+c_{3}\right] \\
& =\frac{1}{N T}\left\{\sum_{j=0}^{N-1}\left[c_{1} \int_{0}^{T} h\left(t+A_{j} T\right) d t+c_{2}\right]+c_{3}-c_{2}\right\},
\end{aligned}
$$

where $h(t) \equiv f(t) / \bar{F}(t), \quad f(t) \equiv d F(t) / d t, \quad \bar{\Phi} \equiv 1-\Phi$, and $A_{j} \equiv \sum_{i=1}^{j} a_{i}$ and $A_{0} \equiv 0(j=$ $1,2, \cdots, N)$ indicates the effectiveness of daily e-learning. $A_{j}$ represents the imperfect rate of maintenance in the reliability model, but in this educational model, it represents the imperfect rate of the reminding effect of trainings. Because the results of trainings and the occurrence of accidents can be measured quantitatively, $A_{j}$ can be calculated by accumulating an actual education data.

\subsection{Optimal Policy 1}

We find optimal $N_{1}^{*}$ to minimize $C_{1}(N, T ; a)$ for a fixed $T$ when $h(t)$ increases strictly with $t$. From the inequality $C_{1}(N+1, T ; a)-C_{1}(N, T ; a) \geq 0$,

$L_{11}(N) \geq \frac{c_{3}-c_{2}}{c_{1}}$

where,

$$
L_{11}(N) \equiv \sum_{j=0}^{N-1} \int_{0}^{T}\left[h\left(t+A_{N} T\right)-h\left(t+A_{j} T\right)\right] d t
$$

Note that

$$
L_{11}(1) \equiv \int_{0}^{T}\left[h\left(t+A_{1} T\right)-h(t)\right] d t
$$

and

$$
L_{11}(N+1)-L_{11}(N) \equiv(N+1) \int_{0}^{T}\left[h\left(t+A_{N+1} T\right)-h\left(t+A_{N} T\right)\right] d t>0 .
$$

Thus, $L_{11}(N)$ increases strictly with $N$ from $L_{11}(1)$ to $L_{11}(\infty) \equiv \lim _{N \rightarrow \infty} L_{11}(N)$. Therefore, we have the following optimum policy:

(i) If $L_{11}(1)<\left(c_{3}-c_{2}\right) / c_{1}$ and $L_{11}(\infty)>\left(c_{3}-c_{2}\right) / c_{1}$ then there exists a finite and unique minimum $N_{1}^{*}\left(1 \leq N_{1}^{*}<\infty\right)$ that satisfies $(2)$.

(ii) If $L_{11}(\infty) \leq\left(c_{3}-c_{2}\right) / c_{1}$ then $N_{1}^{*}=\infty$, i.e., we should not stop the training. 


\subsection{Optimal Policy 2}

We find optimal $T_{1}^{*}$ to minimize $C_{1}(N, T ; a)$ for a fixed $N(1 \leq N<\infty)$ in (1). Differentiating $C_{1}(N, T ; a)$ with respect to $T$ and setting it equal to zero,

$L_{12}(T)=\frac{(N-1) c_{2}+c_{3}}{c_{1}}$

where,

$$
\begin{aligned}
L_{12}(T) \equiv & \sum_{j=0}^{N-1}\left[\left(1+A_{j}\right) \operatorname{Th}\left(\left(1+A_{j}\right) T\right)-A_{j} \operatorname{Th}\left(A_{j} T\right)-\int_{0}^{T} h\left(t+A_{j} T\right) d t\right] \\
& =\sum_{j=0}^{N-1} \int_{0}^{T}\left(t+A_{j} T\right) d h\left(t+A_{j} T\right)=\sum_{j=0}^{N-1} \int_{A_{j} T}^{\left(1+A_{j}\right) T} \operatorname{tdh}(t) .
\end{aligned}
$$

It can be easily seen that $L_{12}(T)$ increases strictly with $T$ from $L_{12}(0)=0$ to $L_{12}(\infty)=\infty$. Therefore, we have the following optimum policy:

(i) If $L_{12}(\infty)>\left((N-1) c_{2}+c_{3}\right) / c_{1}$, then there exists a finite and unique minimum $T_{1}^{*}\left(0<T_{1}^{*}<\infty\right)$ which satisfies $(3)$, and the resulting cost rate is:

$C_{1}\left(N, T_{1}^{*} ; a\right)=\frac{c_{1}}{N} \sum_{j=0}^{N-1}\left[\left(1+A_{j}\right) h\left(\left(1+A_{j}\right) T_{1}^{*}\right)-A_{j} h\left(A_{j} T_{1}^{*}\right)\right]$

(ii) If $L_{12}(\infty) \leq\left((N-1) c_{2}+c_{3}\right) / c_{1}$ then $T_{1}^{*}=\infty$, we should not make the training, and $\lim _{T_{1}^{*} \rightarrow \infty} C_{1}\left(N, T_{1}^{*} ; a\right)=\frac{c_{1}}{N} h(\infty)$

\subsection{Particular Cases}

When $h(t)$ increases strictly with $t$ from $h(0)=0$ to $h(\infty)=\infty$, we consider the following two cases of $A_{j}$ s such that $a_{i}=$ a for case 1 and $A_{j}=\sum_{i=1}^{j} a^{i}$ for case 2 .

Note that $A_{j}$ in case 2 is smaller than that in case 1 and the effectiveness in case 1 is lower than that in case $2 . A_{j}$ in case 1 can be applied to problem solving exercises because the effectiveness of elearnings such as problem solving exercises may be lower than that of classroom lectures (see Sadeghi, 2019).

(1) Case $1: a_{i}=a$

When $a_{i}=\mathrm{a}(0<a<1), A_{j}=j \mathrm{a}(j=0,1, \ldots, N),(2)$ is:

$\sum_{j=0}^{N-1} \int_{0}^{T}[h(t+N a T)-h(t+j a T)] d t \geq \frac{c_{3}-c_{2}}{c_{1}}$

whose left-hand side increases strictly with $N$ from $\int_{0}^{T}[h(t+a T)-h(t)] d t$ to $\infty$. Thus, there exists a finite and unique minimum $N_{11}^{*}\left(1 \leq N_{11}^{*}<\infty\right)$ which satisfies $(6)$. 
Equation (3) is

$\sum_{j=0}^{N-1} \int_{j a T}^{(1+j a) T} t d h(t)=\frac{(N-1) c_{2}+c_{3}}{c_{1}}$

whose left-hand side increases strictly with $T$ from 0 to $\infty$. Thus, there exists a finite and unique $T_{11}^{*}\left(0 \leq T_{11}^{*}<\infty\right)$ which satisfies $(7)$, and the resulting cost rate is:

$C_{1}\left(N, T_{11}^{*} ; a\right)=\frac{c_{1}}{N} \sum_{j=0}^{N-1}\left[(1+j a) h\left((1+j a) T_{11}^{*}\right)-j a h\left(j a T_{11}^{*}\right)\right]$

(2) Case $2: a_{i}=a^{i}$

when $a_{i}=\mathrm{a}^{i}(0<a<1), A_{j}=\sum_{i=1}^{j} a^{i}=\mathrm{a}\left(1-a^{j}\right) /(1-a)(j=0,1, \ldots, N),(2)$ is:

$\sum_{j=0}^{N-1} \int_{0}^{T}\left[h\left(t+\frac{1-a^{N}}{1-a} a T\right)-h\left(t+\frac{1-a^{j}}{1-a} a T\right)\right] d t \geq \frac{c_{3}-c_{2}}{c_{1}}$

whose left-hand side increases strictly with $N$ from $\int_{0}^{T}[h(t+a T)-h(t)] d t$ to $\infty$. Thus, there exists a finite and unique minimum $N_{12}^{*}\left(1 \leq N_{12}^{*}<\infty\right)$ which satisfies (9).

Equation (3) is:

$\sum_{j=0}^{N-1} \int_{0}^{T}\left(t+\frac{1-a^{j}}{1-a} a T\right) d h\left(t+\frac{1-a^{j}}{1-a} a T\right)=\frac{(N-1) c_{2}-c_{3}}{c_{1}}$

whose left-hand side increases strictly with $T$ from 0 to $\infty$. Thus, there exists a finite and unique $T_{12}^{*}\left(0 \leq T_{12}^{*}<\infty\right)$ which satisfies $(10)$, and the resulting cost rate is:

$$
\begin{aligned}
C_{1}\left(N, T_{12}^{*} ; a\right)= & \frac{c_{1}}{N} \sum_{j=0}^{N-1}\left[\left(1+\frac{1-a^{j}}{1-a} a\right) h\left(\left(1+\frac{1-a^{j}}{1-a} a\right) T_{12}^{*}\right)\right. \\
& \left.-\frac{1-a^{j}}{1-a} a h\left(\frac{1-a^{j}}{1-a} a T_{12}^{*}\right)\right]
\end{aligned}
$$

\section{Model 2}

Model 2 is an expanded model of Model 1. Although off-the-job CVET is undergone at time NT in Model 1, the CVET is undergone immediately when a serious accident occurs. We make assumptions 1), 3), 4) of Model 1 and assumption 2') is revised from 2) of the Model 1 as follows:

2') Some accidents related with case studies by forgetting lessons occur during the training. When the exercise volume $T$ is the function of time, its occurrence rate is given by $h(t)$ at time $t$, which remains undisturbed by any accidents and increases strictly with time $t$. Accidents are divided into two categories, i.e., serious ones and not-serious ones. Because serious accidents include the loss of life and the loss of equipment, the contents of safety training are improved and the training is undergone immediately when serious ones happen. The occurrence probability of serious accidents is $q(\equiv 1-p)$ for $0<p<1$. 
When the time from a training to the next training is assumed as one cycle, the mean time of one cycle is:

$\sum_{j=1}^{N} j T p^{j-1} q+N T p^{N}=T \frac{1-p^{N}}{q}$

when $A_{j} \equiv j$, the expected cost of one cycle is, with reference to (see Nakagawa, p179, 2005),

$$
\begin{gathered}
\sum_{j=1}^{N} p^{j-1} q\left[c_{1} \int_{0}^{j T} h(t) d t+j c_{2}\right]+p^{N}\left[c_{1} \int_{0}^{N T} h(t) d t+(N-1) c_{2}+c_{3}\right] \\
=\sum_{j=0}^{N-1} p^{j}\left[c_{1} \int_{j T}^{(j+1) T} h(t) d t+c_{2}\right]+p^{N}\left(c_{3}-c_{2}\right)
\end{gathered}
$$

thus, dividing (13) by (12), the expected cost rate of one cycle is:

$C_{2}(N, T ; p)=\frac{q}{T\left(1-p^{N}\right)}\left\{\sum_{j=0}^{N-1} p^{j}\left[c_{1} \int_{j T}^{(j+1) T} h(t) d t+c_{2}\right]+p^{N}\left(c_{3}-c_{2}\right)\right\}$

when the effectiveness of problem solving exercise $A_{j}$ is considered, (14) is:

$C_{2}(N, T ; p, a)$

$=\frac{q}{T\left(1-p^{N}\right)}\left\{\sum_{j=0}^{N-1} p^{j}\left[c_{1} \int_{A_{j} T}^{\left(1+A_{j}\right) T} h(t) d t+c_{2}\right]+p^{N}\left(c_{3}-c_{2}\right)\right\}$
$=\frac{q}{T\left(1-p^{N}\right)}\left\{\sum_{j=0}^{N-1} p^{j}\left[c_{1} \int_{0}^{T} h\left(t+A_{j} T\right) d t+c_{2}\right]+p^{N}\left(c_{3}-c_{2}\right)\right\}$

which agree with (1) as $p=1$.

\subsection{Optimal Policy 1}

We find optimal $N_{2}^{*}$ to minimize $C_{2}(N, T ; p, a)$ for a fixed $T$. From the inequality $C_{2}(N+$ $1, T ; p, a)-C_{2}(N, T ; p, a) \geq 0$,

$L_{21}(N) \geq \frac{c_{3}-c_{2}}{c_{1}}$

where

$$
L_{21}(N)=\sum_{j=0}^{N-1} p^{j} \int_{0}^{T}\left[h\left(t+A_{N} T\right)-h\left(t+A_{j} T\right)\right] d t
$$

Note that

and

$$
L_{21}(1) \equiv \int_{0}^{T}\left[h\left(t+A_{1} T\right)-h(t)\right] d t
$$


$L_{21}(N+1)-L_{21}(N)=\sum_{j=0}^{N} p^{j} \int_{0}^{T}\left[h\left(t+A_{N+1} T\right)-h\left(t+A_{N} T\right)\right] d t>0$

thus, $L_{21}(N)$ increases strictly with $N$ from $L_{21}(1)$ to $L_{21}(\infty) \equiv \lim _{N \rightarrow \infty} L_{21}(N)$. Therefore, we have the following optimum policy:

(i) If $L_{21}(1)<\left(c_{3}-c_{2}\right) / c_{1}$ and $L_{21}(\infty)>\left(c_{3}-c_{2}\right) / c_{1}$ then there exists a finite and unique minimum $N_{2}^{*}$ that satisfies (16).

(ii) If $L_{21}(\infty) \leq\left(c_{3}-c_{2}\right) / c_{1}$ then $N_{2}^{*}=\infty$, i.e., the training should not be made.

Because $L_{21}(N) \leq L_{11}(N), N_{2}^{*} \geq N_{1}^{*}$.

\subsection{Optimal Policy 2}

We find optimal $T_{2}^{*}$ to minimize $C_{2}(N, T ; p, a)$ for a fixed $N$. Differentiating $C_{2}(N, T ; p, a)$ with respect to $T$ and setting it equal to zero,

$L_{22}(T)=\frac{1}{c_{1}}\left[c_{2} \frac{1-(1+q) p^{N}}{q}+c_{3} p^{N}\right]$

where

$$
L_{22}(T) \equiv \sum_{j=0}^{N-1} p^{j} \int_{0}^{T}\left(t+A_{j} T\right) d h\left(t+A_{j} T\right)=\sum_{j=0}^{N-1} p^{j} \int_{A_{j} T}^{\left(1+A_{j}\right) T} t d h(t)
$$

which is strictly increasing in $T$ from $L_{22}(0)=0$ to $L_{22}(\infty)=\infty$. Therefore, we have the following optimum policy:

(i) If $L_{22}(\infty)>\left\{c_{2}\left[1-(1+q) p^{N}\right] / q+c_{3} p^{N}\right\} / c_{1}$ then there exists a finite and unique minimum $T_{2}^{*}$ that satisfies (18). The resulting cost rate is:

$C_{2}\left(N, T_{2}^{*} ; p, a\right)=\frac{c_{1} q}{1-p^{N}} \sum_{j=0}^{N-1} p^{j}\left[\left(1+A_{j}\right) h\left(\left(1+A_{j}\right) T_{2}^{*}\right)-A_{j} h\left(A_{j} T_{2}^{*}\right)\right]$

(ii) $\quad L_{22}(\infty) \leq\left\{c_{2}\left[1-(1+q) p^{N}\right] / q+c_{3} p^{N}\right\} / c_{1}$ then $T_{2}^{*}=\infty$, i.e., we should not make the training.

\subsection{Particular Cases}

We consider the following two cases of $A_{j}$ 's such that $a_{i}=$ a for case 1 and $A_{j}=\sum_{i=1}^{j} a^{i}$ for case 2.

(1) Case 1: $a_{i}=a$

When $a_{i}=\mathrm{a}(0<a<1), A_{j}=j \mathrm{a}(j=0,1, \ldots, N),(16)$ is:

$\sum_{j=0}^{N-1} p^{j} \int_{0}^{T}[h(t+N a T)-h(t+j a T)] d t \geq \frac{c_{3}-c_{2}}{c_{1}}$

whose left-hand side increases strictly with $N$ from $\int_{0}^{T}[h(t+a T)-h(t)] d t$ to $\infty$. Thus, there 
exists a finite and unique minimum $N_{21}^{*}\left(1 \leq N_{21}^{*}<\infty\right)$ which satisfies (20). Because the left-hand side of (20) is less than the left-hand side of (6), $N_{21}^{*}$ is greater than $N_{11}^{*}$.

Equation (18) is

$\sum_{j=0}^{N-1} p^{j} \int_{j a T}^{(1+j a) T} t d h(t)=\frac{1}{c_{1}}\left[c_{2} \frac{1-(1+q) p^{N}}{q}+c_{3} p^{N}\right]$

whose left-hand side increases strictly with $T$ from 0 to $\infty$. Thus, there exists a finite and unique $T_{21}^{*}\left(0 \leq T_{21}^{*}<\infty\right)$ which satisfies $(21)$, and the resulting cost rate is:

$C_{2}\left(N, T_{21}^{*} ; p, a\right)=\frac{c_{1} q}{1-p^{N}} \sum_{j=0}^{N-1} p^{j}\left[(1+j a) h\left((1+j a) T_{21}^{*}\right)-j a h\left(j a T_{21}^{*}\right)\right]$

(2) Case $2: a_{i}=a^{i}$

When $a_{i}=\mathrm{a}^{i}(0<a<1), A_{j}=\sum_{i=1}^{j} a^{i}=\mathrm{a}\left(1-a^{j}\right) /(1-a)(j=0,1, \ldots, N),(16)$ is:

$\sum_{j=0}^{N-1} p^{j} \int_{0}^{T}\left[h\left(t+\frac{1-a^{N}}{1-a} a T\right)-h\left(t+\frac{1-a^{j}}{1-a} a T\right)\right] d t \geq \frac{c_{3}-c_{2}}{c_{1}}$

whose left-hand side increases strictly with $N$ from $\int_{0}^{T}[h(t+a T)-h(t)] d t$ to $\infty$. Thus, there exists a finite and unique minimum $N_{22}^{*}\left(1 \leq N_{22}^{*}<\infty\right)$ which satisfies (23). Because the left-hand side of (23) is less than the left-hand side of (9), $N_{22}^{*}$ is greater than $N_{12}^{*}$.

Equation (18) is

$\sum_{j=0}^{N-1} p^{j} \int_{0}^{T}\left(t+\frac{1-a^{j}}{1-a} a T\right) d h\left(t+\frac{1-a^{j}}{1-a} a T\right)=\frac{1}{c_{1}}\left[c_{2} \frac{1-(1+q) p^{N}}{q}+c_{3} p^{N}\right]$

whose left-hand side increases strictly with $T$ from 0 to $\infty$. Thus, there exists a finite and unique $T_{22}^{*}\left(0 \leq T_{22}^{*}<\infty\right)$ which satisfies $(24)$, and the resulting cost rate is:

$$
\begin{aligned}
C_{2}\left(N, T_{22}^{*} ; p, a\right) & =\frac{c_{1} q}{1-p^{N}} \sum_{j=0}^{N-1} p^{j}\left[\left(1+\frac{1-a^{j}}{1-a} a\right) h\left(\left(1+\frac{1-a^{j}}{1-a} a\right) T_{22}^{*}\right)\right. \\
& \left.-\frac{1-a^{j}}{1-a} a h\left(\frac{1-a^{j}}{1-a} a T_{22}^{*}\right)\right]
\end{aligned}
$$

\section{Numerical Illustrations}

We assume that $F(t)=1-\exp \left(-\lambda t^{m}\right)$ and $h(t)=m \lambda t^{m-1}$ because $F(t)$ is strict increasing with $t$ and the exponential distribution is widely used in past studies. When $c_{1}=1, c_{2}=2, c_{3}=$ 10 , and $A_{j}=j a$, optimal $T_{11}^{*}$ and $C_{1}\left(N, T_{11}^{*} ; a\right)$ are shown in Table 1 . When $\lambda, m, N$, and $a$ increase, $T_{11}^{*}$ decreases and $C_{1}\left(N, T_{11}^{*} ; a\right)$ increases. 
Table 1. Optimal $T_{11}^{*}$ and resulting cost rate $C_{1}\left(N, T_{11}^{*} ; a\right)$ when $c_{1}=1, c_{2}=2, c_{3}=10$, and $A_{j}=j a$.

\begin{tabular}{|c|c|c|c|c|c|}
\hline $\boldsymbol{\lambda}$ & $\boldsymbol{m}$ & $\boldsymbol{N}$ & $\boldsymbol{a}$ & $\boldsymbol{T}_{\mathbf{1 1}}^{*}$ & $\boldsymbol{C}_{\mathbf{1}}\left(\boldsymbol{N}, \boldsymbol{T}_{11}^{*} ; \boldsymbol{a}\right)$ \\
\hline 1 & 1.2 & 10 & 0.95 & 6.188 & 2.715 \\
\hline 2 & 1.2 & 10 & 0.95 & 3.473 & 6.838 \\
\hline 3 & 1.2 & 10 & 0.95 & 2.477 & 6.783 \\
\hline 1 & 1.25 & 10 & 0.95 & 4.396 & 3.184 \\
\hline 1 & 1.3 & 10 & 0.95 & 3.309 & 2.666 \\
\hline 1 & 1.2 & 8 & 0.95 & 6.800 & 2.569 \\
\hline 1 & 1.2 & 6 & 0.95 & 7.785 & 2.723 \\
\hline 1 & 1.2 & 10 & 0.97 & 6.170 & 2.731 \\
\hline 1 & 1.2 & 10 & 0.99 & 6.152 & \\
\hline
\end{tabular}

Table 2 presents optimal $T_{12}^{*}$ and $C_{1}\left(N, T_{12}^{*} ; a\right)$ when $c_{1}=1, c_{2}=2, c_{3}=10$, and $A_{j}=\sum_{i=1}^{j} a^{i}$. When $\lambda, m, N$, and $a$ increase, $T_{12}^{*}$ decreases and $C_{1}\left(N, T_{12}^{*} ; a\right)$ increases which are similar to Table 1. $T_{12}^{*}$ and $1 / C_{1}\left(N, T_{12}^{*} ; a\right)$ of Table 2 are greater than those of Table 1.

Table 2. Optimal $T_{12}^{*}$ and resulting cost rate $C_{1}\left(N, T_{12}^{*} ; a\right)$ when $c_{1}=1, c_{2}=2, c_{3}=10$, and $A_{j}=\sum_{i=1}^{j} a^{i}$.

\begin{tabular}{|c|c|c|c|c|c|}
\hline $\boldsymbol{\lambda}$ & $\boldsymbol{m}$ & $\boldsymbol{N}$ & $\boldsymbol{a}$ & $\boldsymbol{T}_{\mathbf{1 2}}^{*}$ & $\boldsymbol{C}_{\mathbf{1}}\left(\boldsymbol{N}, \boldsymbol{T}_{\mathbf{1 2}}^{*} ; \boldsymbol{a}\right)$ \\
\hline 1 & 1.2 & 10 & 0.95 & 6.282 & 2.674 \\
\hline 2 & 1.2 & 10 & 0.95 & 3.526 & 6.765 \\
\hline 3 & 1.2 & 10 & 0.95 & 2.515 & 6.681 \\
\hline 1 & 1.25 & 10 & 0.95 & 4.479 & 3.126 \\
\hline 1 & 1.3 & 10 & 0.95 & 3.383 & 3.587 \\
\hline 1 & 1.2 & 8 & 0.95 & 6.875 & 2.618 \\
\hline 1 & 1.2 & 6 & 0.95 & 7.839 & 2.551 \\
\hline 1 & 1.2 & 10 & 0.97 & 6.227 & 2.698 \\
\hline 1 & 1.2 & 10 & 0.99 & 6.171 & 2.722 \\
\hline
\end{tabular}

When $c_{1}=1, c_{2}=2, c_{3}=10$, and $A_{j}=j a$, optimal $N_{11}^{*}$ and $C_{1}\left(N_{11}^{*}, T ; a\right)$ are shown in Table 3 . When $\lambda$ and $m$ increase, $N_{11}^{*}$ decreases and $C_{1}\left(N_{11}^{*}, T ; a\right)$ increases, and $N_{11}^{*}$ and $C_{1}\left(N_{11}^{*}, T ; a\right)$ decrease when $T$ increases. $N_{11}^{*}$ does not change and $C_{1}\left(N_{11}^{*}, T ; a\right)$ increases when $a$ increases in this numerical illustration.

Table 3. Optimal $N_{11}^{*}$ and resulting cost rate $C_{1}\left(N_{11}^{*}, T ; a\right)$ when $c_{1}=1, c_{2}=2, c_{3}=10$, and $A_{j}=j a$.

\begin{tabular}{|c|c|c|c|c|c|}
\hline $\boldsymbol{\lambda}$ & $\boldsymbol{m}$ & $\boldsymbol{T}$ & $\boldsymbol{a}$ & $\boldsymbol{N}_{\mathbf{1 1}}^{*}$ & $\boldsymbol{C}_{\mathbf{1}}\left(\boldsymbol{N}_{\mathbf{1 1}}^{*}, \boldsymbol{T} ; \boldsymbol{a}\right)$ \\
\hline 1 & 1.2 & 2 & 0.95 & 6 & 3.203 \\
\hline 2 & 1.2 & 2 & 0.95 & 4 & 6.929 \\
\hline 3 & 1.2 & 2 & 0.95 & 8 & 3.480 \\
\hline 1 & 1.25 & 2 & 0.95 & 6 & 3.750 \\
\hline 1 & 1.3 & 2 & 0.95 & 6 & 2.707 \\
\hline 1 & 1.2 & 4 & 0.95 & 4 & 2.543 \\
\hline 1 & 1.2 & 6 & 0.95 & 11 & 3.210 \\
\hline 1 & 1.2 & 2 & 0.97 & 11 & 3.216 \\
\hline 1 & 1.2 & 2 & 0.99 & & \\
\hline
\end{tabular}

Table 4 presents optimal $N_{12}^{*}$ and $C_{1}\left(N_{12}^{*}, T ; a\right)$ when $c_{1}=1, c_{2}=2, c_{3}=10$, and $A_{j}=\sum_{i=1}^{j} a^{i}$. When $\lambda$ and $m$ increase, $N_{12}^{*}$ decreases and $C_{1}\left(N_{12}^{*}, T ; a\right)$ increases, and $N_{12}^{*}$ and $C_{1}\left(N_{12}^{*}, T ; a\right)$ decrease when $T$ increases which are similar to Table $3 . N_{12}^{*}$ decreases and $C_{1}\left(N_{12}^{*}, T ; a\right)$ increases 
when $a$ increases. $N_{12}^{*}$ and $1 / C_{1}\left(N_{12}^{*}, T ; a\right)$ of Table 4 are greater than or are equal to those of Table 3.

Table 4. Optimal $N_{12}^{*}$ and resulting cost rate $C_{1}\left(N_{12}^{*}, T ; a\right)$ when $c_{1}=1, c_{2}=2, c_{3}=10$, and $A_{j}=$ $\sum_{i=1}^{j} a^{i}$.

\begin{tabular}{|c|c|c|c|c|c|}
\hline $\boldsymbol{\lambda}$ & $\boldsymbol{m}$ & $\boldsymbol{T}$ & $\boldsymbol{a}$ & $\boldsymbol{N}_{\mathbf{1 2}}^{*}$ & $\boldsymbol{C}_{\mathbf{1}}\left(\boldsymbol{N}_{\mathbf{1 2}}^{*}, \boldsymbol{T} ; \boldsymbol{a}\right)$ \\
\hline 1 & 1.2 & 2 & 0.95 & 13 & 3.161 \\
\hline 2 & 1.2 & 2 & 0.95 & 7 & 6.899 \\
\hline 3 & 1.2 & 2 & 0.95 & 5 & 3.493 \\
\hline 1 & 1.25 & 2 & 0.95 & 9 & 3.442 \\
\hline 1 & 1.3 & 2 & 0.95 & 7 & 2.692 \\
\hline 1 & 1.2 & 4 & 0.95 & 6 & 2.536 \\
\hline 1 & 1.2 & 6 & 0.95 & 4 & 3.185 \\
\hline 1 & 1.2 & 2 & 0.97 & 12 & 3.208 \\
\hline 1 & 1.2 & 2 & 0.99 & 11 & \\
\hline
\end{tabular}

When $c_{1}=1, c_{2}=2, c_{3}=10$, and $A_{j}=j a$, optimal $T_{21}^{*}$ and $C_{2}\left(N, T_{21}^{*} ; p, a\right)$ are shown in Table 5. When $\lambda, m, N$, and $a$ increase, $T_{21}^{*}$ decreases and $C_{2}\left(N, T_{21}^{*} ; p, a\right)$ increases which are similar to Table 1 , and $T_{21}^{*}$ and $C_{2}\left(N, T_{21}^{*} ; p, a\right)$ increase when $p$ increases. $T_{21}^{*}$ and $C_{2}\left(N, T_{21}^{*} ; p, a\right)$ in Table 5 are smaller than $T_{11}^{*}$ and $C_{1}\left(N, T_{11}^{*} ; a\right)$ in Table 1.

Table 5. Optimal $T_{21}^{*}$ and resulting cost rate $C_{2}\left(N, T_{21}^{*} ; p, a\right)$ when $c_{1}=1, c_{2}=2, c_{3}=10$, and $A_{j}=j a$.

\begin{tabular}{|c|c|c|c|c|c|c|}
\hline$\lambda$ & $m$ & $N$ & $a$ & $p$ & $T_{21}^{*}$ & $C_{2}\left(N, T_{21}^{*} ; p, a\right)$ \\
\hline 1 & 1.2 & 10 & 0.95 & 0.90 & 5.724 & 2.545 \\
\hline 2 & 1.2 & 10 & 0.95 & 0.90 & 3.212 & 4.535 \\
\hline 3 & 1.2 & 10 & 0.95 & 0.90 & 2.291 & 6.359 \\
\hline 1 & 1.25 & 10 & 0.95 & 0.90 & 4.116 & 2.950 \\
\hline 1 & 1.3 & 10 & 0.95 & 0.90 & 3.132 & 3.360 \\
\hline 1 & 1.2 & 8 & 0.95 & 0.90 & 6.242 & 2.504 \\
\hline 1 & 1.2 & 6 & 0.95 & 0.90 & 7.111 & 2.453 \\
\hline 1 & 1.2 & 10 & 0.97 & 0.90 & 5.708 & 2.552 \\
\hline 1 & 1.2 & 10 & 0.99 & 0.90 & 5.693 & 2.559 \\
\hline 1 & 1.2 & 10 & 0.95 & 0.85 & 5.585 & 2.464 \\
\hline 1 & 1.2 & 10 & 0.95 & 0.80 & 5.508 & 2.388 \\
\hline
\end{tabular}

Table 6 presents optimal $T_{22}^{*}$ and $C_{2}\left(N, T_{22}^{*} ; p, a\right)$ when, $c_{1}=1, c_{2}=2, c_{3}=10$, and $A_{j}=$ $\sum_{i=1}^{j} a^{i}$. When $\lambda, m, N$, and $a$ increase, $T_{22}^{*}$ decreases and $C_{2}\left(N, T_{22}^{*} ; p, a\right)$ increases, and $T_{22}^{*}$ and $C_{2}\left(N, T_{22}^{*} ; p, a\right)$ increase when $p$ increases which are similar to Table $5 . T_{22}^{*}$ and $1 / C_{2}\left(N, T_{22}^{*} ; p, a\right)$ of Table 6 are greater than those of Table $5 . T_{22}^{*}$ and $C_{2}\left(N, T_{22}^{*} ; p, a\right)$ in Table 6 are smaller than $T_{12}^{*}$ and $C_{1}\left(N, T_{12}^{*} ; a\right)$ in Table 2. 
Table 6. Optimal $T_{22}^{*}$ and resulting cost rate $C_{2}\left(N, T_{22}^{*} ; p, a\right)$ when $c_{1}=1, c_{2}=2, c_{3}=10$, and $A_{j}=$ $\sum_{i=1}^{j} a^{i}$.

\begin{tabular}{|c|c|c|c|c|c|c|}
\hline $\boldsymbol{\lambda}$ & $\boldsymbol{m}$ & $\boldsymbol{N}$ & $\boldsymbol{a}$ & $\boldsymbol{p}$ & $\boldsymbol{T}_{\mathbf{2 2}}^{*}$ & $\boldsymbol{C}_{\mathbf{2}}\left(\boldsymbol{N}, \boldsymbol{T}_{\mathbf{2 2}}^{*} ; \boldsymbol{p}, \boldsymbol{a}\right)$ \\
\hline 1 & 1.2 & 10 & 0.95 & 0.90 & 5.794 & 2.515 \\
\hline 2 & 1.2 & 10 & 0.95 & 0.90 & 3.252 & 4.481 \\
\hline 3 & 1.2 & 10 & 0.95 & 0.90 & 2.319 & 6.282 \\
\hline 1 & 1.25 & 10 & 0.95 & 0.90 & 4.178 & 2.906 \\
\hline 1 & 1.3 & 10 & 0.95 & 0.90 & 3.188 & 3.301 \\
\hline 1 & 1.2 & 8 & 0.95 & 0.90 & 6.299 & 2.481 \\
\hline 1 & 1.2 & 6 & 0.95 & 0.90 & 7.153 & 2.439 \\
\hline 1 & 1.2 & 10 & 0.97 & 0.90 & 5.750 & 2.534 \\
\hline 1 & 1.2 & 10 & 0.99 & 0.90 & 5.707 & 2.553 \\
\hline 1 & 1.2 & 10 & 0.95 & 0.85 & 5.645 & 2.439 \\
\hline 1 & 1.2 & 10 & 0.95 & 0.80 & 5.558 & 2.367 \\
\hline
\end{tabular}

When $c_{1}=1, c_{2}=2, c_{3}=10$, and $A_{j}=j a$, optimal $N_{21}^{*}$ and $C_{2}\left(N_{21}^{*}, T ; p, a\right)$ are shown in Table 7. When $\lambda$ and $m$ increase, $N_{21}^{*}$ decreases and $C_{2}\left(N_{21}^{*}, T ; p, a\right)$ increases, and $N_{21}^{*}$ and $C_{2}\left(N_{21}^{*}, T ; p, a\right)$ decrease when $T$ increases which are similar to Table 3. $N_{21}^{*}$ does not change and $C_{2}\left(N_{21}^{*}, T ; p, a\right)$ increases when $a$ increases which are similar to Table 3 . When $p$ increases, $N_{21}^{*}$ decreases and $C_{2}\left(N_{21}^{*}, T ; p, a\right)$ increases. $N_{21}^{*}$ and $1 / C_{2}\left(N_{21}^{*}, T ; p, a\right)$ of Table 7 are greater than or are equal to $N_{11}^{*}$ and $1 / C_{1}\left(N_{11}^{*}, T ; a\right)$ of Table 3 .

Table 7. Optimal $N_{21}^{*}$ and resulting cost rate $C_{2}\left(N_{21}^{*}, T ; p, a\right)$ when $c_{1}=1, c_{2}=2, c_{3}=10$, and $A_{j}=j a$.

\begin{tabular}{|c|c|c|c|c|c|c|}
\hline$\lambda$ & $m$ & $T$ & $a$ & $p$ & $N_{21}^{*}$ & $C_{2}\left(N_{21}^{*}, T ; p, a\right)$ \\
\hline 1 & 1.2 & 2 & 0.95 & 0.90 & 14 & 2.918 \\
\hline 2 & 1.2 & 2 & 0.95 & 0.90 & 7 & 4.620 \\
\hline 3 & 1.2 & 2 & 0.95 & 0.90 & 5 & 6.191 \\
\hline 1 & 1.25 & 2 & 0.95 & 0.90 & 10 & 3.184 \\
\hline 1 & 1.3 & 2 & 0.95 & 0.90 & 7 & 3.445 \\
\hline 1 & 1.2 & 4 & 0.95 & 0.90 & 6 & 2.549 \\
\hline 1 & 1.2 & 6 & 0.95 & 0.90 & 4 & 2.431 \\
\hline 1 & 1.2 & 2 & 0.97 & 0.90 & 14 & 2.924 \\
\hline 1 & 1.2 & 2 & 0.99 & 0.90 & 14 & 2.930 \\
\hline 1 & 1.2 & 2 & 0.95 & 0.85 & 17 & 2.798 \\
\hline 1 & 1.2 & 2 & 0.95 & 0.80 & 21 & 2.698 \\
\hline
\end{tabular}

Table 8 presents optimal $N_{22}^{*}$ and $C_{2}\left(N_{22}^{*}, T ; p, a\right)$ when $c_{1}=1, c_{2}=2, c_{3}=10$, and $A_{j}=$ $\sum_{i=1}^{j} a^{i}$. When $\lambda, m$, and $a$ increase, $N_{22}^{*}$ decreases and $C_{2}\left(N_{22}^{*}, T ; p, a\right)$ increases, and $N_{22}^{*}$ and $C_{2}\left(N_{22}^{*}, T ; p, a\right)$ decrease when $T$ increases which are similar to Table 4 . When $p$ increases, $N_{22}^{*}$ decreases and $C_{2}\left(N_{22}^{*}, T ; p, a\right)$ increases which are similar to Table $7 . N_{22}^{*}$ and $1 / C_{2}\left(N_{22}^{*}, T ; p, a\right)$ of Table 8 are greater than or are equal to $N_{12}^{*}$ and $1 / C_{1}\left(N_{12}^{*}, T ; a\right)$ of Table 4.

The validity of the model is shown by confirming that two models and the actual phenomena which are predicted intuitively match by the sensitivity analysis. In order to apply it to real problems, estimating $F(t)$ from actual data is needed. 
Table 8. Optimal $N_{22}^{*}$ and resulting cost rate $C_{2}\left(N_{22}^{*}, T ; p, a\right)$ when $c_{1}=1, c_{2}=2, c_{3}=10$, and $A_{j}=$ $\sum_{i=1}^{j} a^{i}$.

\begin{tabular}{|c|c|c|c|c|c|c|}
\hline $\boldsymbol{\lambda}$ & $\boldsymbol{m}$ & $\boldsymbol{T}$ & $\boldsymbol{a}$ & $\boldsymbol{p}$ & $\boldsymbol{N}_{\mathbf{2 2}}^{*}$ & $\boldsymbol{C}_{\mathbf{2}}\left(\boldsymbol{N}_{\mathbf{2 2}}^{*}, \boldsymbol{T} ; \boldsymbol{p}, \boldsymbol{a}\right)$ \\
\hline 1 & 1.2 & 2 & 0.95 & 0.90 & 19 & 2.875 \\
\hline 2 & 1.2 & 2 & 0.95 & 0.90 & 8 & 4.590 \\
\hline 3 & 1.2 & 2 & 0.95 & 0.90 & 5 & 6.167 \\
\hline 1 & 1.25 & 2 & 0.95 & 0.90 & 11 & 3.146 \\
\hline 1 & 1.3 & 2 & 0.95 & 0.90 & 8 & 3.412 \\
\hline 1 & 1.2 & 4 & 0.95 & 0.90 & 7 & 2.535 \\
\hline 1 & 1.2 & 6 & 0.95 & 0.90 & 4 & 2.425 \\
\hline 1 & 1.2 & 2 & 0.97 & 0.90 & 16 & 2.899 \\
\hline 1 & 1.2 & 2 & 0.99 & 0.90 & 15 & 2.922 \\
\hline 1 & 1.2 & 2 & 0.95 & 0.85 & 27 & 2.760 \\
\hline 1 & 1.2 & 2 & 0.95 & 0.80 & 57 & 2.669 \\
\hline
\end{tabular}

\section{Conclusions}

In this paper, we consider two optimal employee safety education models which minimize the economical investment. In Models 1 and 2, the whole e-learning problem solving time is $T$ and the safety training is performed at $N T$. The safety training is performed immediately when serious accidents happen at occurrence probability $q$ in Model 2. The expected cost rates are defined and optimal $N^{*}$ and $T^{*}$ which minimize the cost rates are discussed and are calculated numerically.

From the numerical illustrations, when the effectiveness of problem solving exercise is improved, i.e., $a$ decreases or $A_{j}$ of case 2 is used, the whole e-learning problem solving time $T^{*}$ and the safety training time $N^{*}$ become great, i.e., the daily e-learning problem solving effort can be reduced because the number of whole problems is constant, and the training time can be prolonged. It shows that the economic investment can be declined by performing the effective daily e-learning.

In this paper, the optimal time interval of safety education and the optimal number of times of elearning to periodical education are discussed when the oblivion of knowledge is approximated by an elementary function and the reliability model is applied. Although the forgetting of safety knowledge is expressed by an elementary function in this paper, the actual forgetting function which is approximated from the actual data would be a complicated one. Today, metaheuristic methods are used for various kinds of complex optimization problems which need to find maximum and minimum values because they can be applicable with few constraints.

Pant et al. (2017a) applied the modified particle swarm optimization (MPSO) algorithm to optimize the reliability of a complex mixed series parallel system. Kumar et al. (2019) applied multi objective gray wolf optimizer (MOGWO) algorithm which was proposed by Mirjalili et al. (2014) to optimize safety designs of nuclear power plants and showed the usefulness of algorithm by comparing it with MOPSO. Kumar et al. applied Cuckoo Search Algorithm (CSA) to optimize a complex bridge system and a life support system (LSS) in space capsule (2017) and applied MOGWO to optimize a LSS in space capsule (2019). Pant et al. (2019) represented a framework devoted to the solution of nonlinear systems of equations using grey wolf optimization algorithm (GWO) and a multi-objective particle swarm optimization algorithm (MOPSO). Negi et al. (2021) reviewed various kinds of applications of the GWO. Pant et al. (2017b) presents modified Flower pollination algorithm (FPA) and applied it to various optimization problems. Uniyal et al. (2020) surveyed nature inspired algorithms and their multi objective optimization applications. Ram et al. 
(2018) summarized various aspects related to complex system reliability analysis and optimization by various kinds of methods including MOPSO. In case of actual data analysis, above metaheuristic methods can be useful.

\section{Conflict of Interest}

The authors confirm that there is no conflict of interest to declare for this publication.

Acknowledgments

This work is supported by Mitsubishi Heavy Industries, LTD.

\section{References}

Aguinis, H., \& Kraiger, K. (2009). Benefits of training and development for individuals and teams, organizations, and society. Annual Review of Psychology, 60, 451-474.

Arago'n-Sa'nchez, A., Barba-Arago'n, I., \& Sanz-Valle, R. (2003). Effects of training on business results. The International Journal of Human Resource Management, 14(6), 956-980.

Aragon, I.B., \& Valle, R.S. (2013). Does training managers pay off?. The International Journal of Human Resource Management. 24(8), 1671-1684. https://doi.org/10.1080/09585192.2012.725064.

Armstrong, B.G., \& Sloan, M. (1989). Ordinal regression models for epidemiologic data. American Journal of Epidemiology, 129(1), 191-204. https://doi.org/10.1093/oxfordjournals.aje.a115109.

Cedefop. (2008). Validation of non-formal and informal learning in europe: a snapshot 2007. Luxembourg: Office for Official Publications of the European Communities. http://www.cedefop.europa.eu/EN/Files/4073_en.pdf.

Cedefop. (2011). The benefits of vocational education and training. Luxembourg, Publications Office of the European Union.

Elshamy, M. (2002). Stochastic models of human errors. NASA/CR-2002-211169.

Gnoni, M.G., \& Saleh, J.H. (2017). Near-miss management systems and observability-in-depth: handling safety incidents and accident precursors in light of safety principles. Safety Science, 91, 154-167.

Harashima, S., Oikawa, D., \& Nihira, H. (2006). Skill enhancement education for software engineers. Toshiba Review, 61(1), 40-43.

Heinrich, H.W. (1931). Industrial accident prevention: A scientific approach. Tata McGraw-Hill Education, New York.

Hickman, J.S., \& Hanowski, R.J. (May 2011). Use of a video monitoring approach to reduce at-risk driving behaviors in commercial vehicle operations. Transportation Research Part F: Traffic Psychology and Behaviour, 14(3), 189-198.

Jaber, M.Y., Givi, Z.S., \& Neumann, W.P. (2013). Incorporating human fatigue and recovery into the learning-forgetting process. Applied Mathematical Modelling, 37(12-13), 7287-7299.

Japan Industrial Safety \& Health Association (JISHA). (2006, May). General guidebook on industrial safety. ILO-CIS National Center in Japan.

Kirkpatrick, D. (1996). Great ideas revisited. techniques for evaluating training programs. revisiting kirkpatrick's four-level model. Training and Development, 50(1), 54-59. 
Kumar, A., Pant, S., \& Ram, M. (2019). Gray wolf optimizer approach to the reliability-cost optimization of residual heat removal system of a nuclear power plant safety system. Quality and Reliability Engineering International, 35(3), 2228-2239.

Kumar, A., Pant, S., \& Singh, S.B. (2017). Reliability optimization of complex system using cuckoos search algorithm. Mathematical Concepts and Applications in Mechanical Engineering and Mechatronics. IGI Global Publisher, 94-110.

Kumar, A., Pant, S., Ram, M. \& Chaube, S. (2017). Multi-objective grey wolf optimizer approach to the reliability-cost optimization of life support system in space capsule. International Journal of System Assurance Engineering and Management, 10, 276-284. https://doi.org/10.1007/s13198-019-00781-1.

Manuele, F.A. (October, 2011). Reviewing heinrich: dislodging two myths from the practice of safety. Professional Safety, 56(10), 52-61.

Maršíková, K., \& Šlaichová, E. (2015). Perspectives of employee training and development: methods and approachee. ACC Journal, 21(3), 13-23. https://doi.org/10.15240/tul/004/2015-3-002.

Masimore, L. (2007). Proving the value of safety: justification and roi of safety programs and machine safety investments. Rockwell Automation, USA.

McGehee, D.V., Raby, M., Carney, C., Lee, J.D., \& Reyes, M.L. (2007). Extending parental mentoring using an event-triggered video intervention in rural teen drivers. Journal of Safety Research, 38(2), 215-227.

Mirjalili, S., Mirjalili, S.M., \& Lewis, A. (2014). Grey wolf optimizer. Advances in Engineering Software, 69, 46-61. https://doi.org/10.1016/j.advengsoft.2013.12.007.

Nakagawa, T. (2005). Maintenance theory of reliability. Springer Verlag, London.

Negi, G., Kumar, A., Pant, S., \& Ram, M. (2021). GWO: a review and applications. International Journal of System Assurance Engineering and Management, 12, 1-8. https://doi.org/10.1007/s13198-020-00995-8.

Pant, S., Kumar, A., \& Ram, M. (2017a). Flower pollination algorithm development: a state of art review. International Journal of System Assurance Engineering and Management. 8(2), 1858-1866.

Pant, S., Kumar, A., \& Ram, M. (2019). Solution of nonlinear systems of equations via metaheuristics. International Journal of Mathematical, Engineering and Management Sciences, 4(5), 1108-1126.

Pant, S., Kumar, A., Singh, S.B., \& Ram, M. (2017b). A modified particle swarm optimization algorithm for nonlinear optimization. Nonlinear Studies, 24(1), 127-138.

Ram, M. \& Davim, J.P. (2018). Complex system reliability analysis and optimization. Advanced Mathematical Techniques in Science and Engineering. River Publishers, 2018, 185-199.

Reason, J. (2000, March 18). Human error: models and management. 320(7237), 768-770. https://doi.org/10.1136/bmj.320.7237.768.

Reason, J., Manstead, A., Stradling, S., Baxter, J., \& Campbell, K. (1990). Errors and violations on the roads: a real distinction?. Ergonomics, 33(10-11), 1315-1332. https://doi.org/10.1080/00140139008925335.

Sadeghi, M. (2019). A shift from classroom to distance learning: advantages and limitations. International Journal of Research in English Education, 4(1), 80-88. URL: http://ijreeonline.com/article-1-132en.html.

Shi, X., Wang, K., \& Xia, T. (2010). Exploring safety education effectiveness on accident risk reduction for freight drivers based on behavioral self-evaluation. International Conference of Logistics Engineering and Management (ICLEM) 2010, pp.1711-1717. Shanghai, China. DOI:10.1061/41139(387)236.

Smidt, A., Balandin, S., Sigafoos, J., \& Reed, V.A. (2009, September). The Kirkpatrick model: a useful tool for evaluating training outcomes. Journal of Intellectual \& Developmental Disability, 34(3), 266-274. 
Topolšek, D., Babić, D., \& Fiolić, M. (March, 2019). The effect of road safety education on the relationship between driver's errors, violations and accidents: slovenian case study. European Transport Research Review, 11(1), 1-8.

Uniyal, N., Pant, S., \& Kumar, A. (2020). An overview of few nature inspired optimization techniques and its reliability applications. International Journal of Mathematical, Engineering and Management Sciences, 5(4), 732-743.

Wang, X., Xing, Y., Luo, L., \& Yu, R. (2018). Evaluating the effectiveness of behavior-based safety education methods for commercial vehicle drivers. Accident Analysis and Prevention, 117, 114-120.

Wani, M. (2007). Technical education for young engineers at Mitsubishi heavy industries. Journal of Japanese Society for Engineering Education, 55(3), 3_17-3_20. https://doi.org/10.4307/jsee.55.3_17. 